\section{Repercusión de la inmunoterapia con alergenos luego de dos años de suspensión a pacientes con asma}

Jorge Sánchez, ${ }^{1,2}$ Ricardo Cardona, ${ }^{1}$ Andrés Sánchez ${ }^{3}$

\section{Resumen}

ANTECEDENTES: la inmunoterapia ha demostrado su eficacia en el control del asma alérgica; sin embargo, pocos estudios han evaluado si el control y la reducción del tratamiento farmacológico persisten al suspenderlo.

OBJETIVO: evaluar el efecto de la inmunoterapia con ácaros en pacientes con asma luego de dos años de suspensión.

MÉTODOS: estudio ambispectivo, observacional, abierto con grupo activo (inmunoterapia y farmacoterapia) y grupo control (farmacoterapia) con seguimiento durante cinco años: dividido en dos fases: tres años de aplicación de la inmunoterapia y dos años de seguimiento luego de su suspensión.

RESULTADOS: se incluyeron 122 pacientes en el grupo activo y 384 en el grupo control. Ambos grupos tuvieron menor número de exacerbaciones luego del sexto mes $(p=0,04)$. Después de nueve meses el grupo activo tuvo una reducción importante en la necesidad de aplicación de esteroides inhalados $(p=0.05)$ versus el grupo control. Después de dos años de la suspensión de la inmunoterapia, la dosis de inhaladores que recibían los pacientes en el grupo activo fue menor que la del grupo control. Los menores de 14 años monosensibilizados tuvieron la mejor respuesta en todos los parámetros evaluados.

CONCLUSIÓN: la inmunoterapia con alergenos disminuye los síntomas del asma y las dosis de medicamentos necesarias para el control del paciente. Estos efectos tienen una repercusión significativa en la calidad de vida y en la economía de los pacientes con asma. El inicio a edades tempranas parece tener mayor impacto clínico.

PALABRAS CLAVE: alergia, asma, ácaros, alérgenos, Dermatophagoides, Blomia, eficacia, costo, economía, inmunoterapia, sensibilización.

Rev Alerg Méx 2016 Apr-Jun;63(2):113-122.

\section{Impact of allergen immunotherapy after two years of suspension in patients with asthma}

Jorge Sánchez, ${ }^{1,2}$ Ricardo Cardona, ${ }^{1}$ Andrés Sánchez ${ }^{3}$

\section{Abstract}

BACKGROUND: Immunotherapy has proven to be effective in controlling allergic asthma. However, few studies have evaluated whether the control and reduction of drug treatment persist after treatment ends.

\footnotetext{
1 Grupo de Alergia Clínica y Experimental. IPS Universitaria, Universidad de Antioquia, Medellín, Antioquia, Colombia.

${ }^{2}$ Fundación para el Desarrollo de las Ciencias Médicas y Biológicas, Cartagena, Colombia.

${ }^{3}$ Corporación Universitaria Rafael Núñez. Programa de Medicina, Cartagena, Colombia.

Recibido: 20 de junio 2015

Aceptado: 22 de octubre 2015

Correspondencia

Jorge Sánchez

jotamsc@yahoo.com

Este artículo debe citarse como

Sánchez J, Cardona R, Sánchez A. Repercusión de la inmunoterapia con alergenos luego de dos años de suspensión a pacientes con asma. Rev Alerg Méx. 2016 abr-jun;63(2):113-122.
} 
OBJECTIVE: To evaluate the effect of immunotherapy with mites in asthma patients after two years of its suspension on drug treatment and number of exacerbations.

MATERIAL AND METHOD: Observational study ambispective with active group (immunotherapy and pharmacotherapy) and control (only pharmacotherapy) group followed up for 5 years divided into two phases: 3 years of application of immunotherapy and two year of follow-up after its suspension.

RESULTS: 122 patients in the active group and 384 in the control group were included. Both groups had fewer exacerbations after the sixth month $(p=0.04)$. After nine months the active group had a significant reduction in the use of inhaled steroids $(p=0.05)$ compared to the control group. Two years after finished immunotherapy, patients in the active group received a lower inhaler doses than the control group. Children under 14 years mono-sensitized had the best response in all parameters evaluated.

CONCLUSION: The allergen immunotherapy improves asthma control and reduces the required doses of pharmacotherapy. These effects have an important impact on quality of life and perhaps economically for patients with asthma. The beginning at an early age seems to have a major impact.

KEY WORDS: Allergy, Asthma, Mites, Allergen, Blomia, Dermatophagoides, Efficacy, Cost, Economy, Immunotherapy, Sensitization.

\author{
${ }^{1}$ Grupo de Alergia Clínica y Experimental. \\ IPS Universitaria, Universidad de Antioquia, \\ Medellín, Antioquia, Colombia. \\ 2 Fundación para el Desarrollo de las \\ Ciencias Médicas y Biológicas, Cartagena, \\ Colombia. \\ ${ }^{3}$ Corporación Universitaria Rafael Núñez. \\ Programa de Medicina, Cartagena, Co- \\ lombia. \\ Correspondence \\ Jorge Sánchez \\ jotamsc@yahoo.com
}

\section{ANTECEDENTES}

El asma es una enfermedad frecuente en Latinoamérica y en la población mundial. ${ }^{1-5}$ En 60 a $80 \%$ de los casos existe un componente atópico en su fisiopatología, con una o varias fuentes de alérgenos como causa de los síntomas. ${ }^{6-9}$ Casi siempre se inicia durante la primera década de la vida y aunque en un grupo importante de pacientes suele remitir durante la adolescencia, muchos continúan con los síntomas y tiende a empeorar con el transcurrir de los años. ${ }^{10}$ Actualmente los medicamentos controladores, como los esteroides y los beta-agonistas, son la piedra angular del tratamiento sintomático del asma, pero no detienen la progresión de nuevas sensibilizaciones y tienen el riesgo de producir efectos adversos con su uso continuo. La inmunoterapia con alérgenos ha demostrado su eficacia en el tratamiento de las enfermedades respiratorias alérgicas ${ }^{11,12}$ pudiendo controlar los síntomas y reducir la necesidad de medicamentos por medio de la inducción de una tolerancia inmunológica a los alérgenos que reacciona el paciente. ${ }^{11,13-15}$ Debido al riesgo de reacciones durante su administración es necesario que el paciente con asma tenga un control sintomático para evitar reacciones severas mientras se consigue su inmunomodulación. ${ }^{16}$ Con el transcurrir del tiempo el paciente consigue un mejor control sintomático y puede tolerar la suspensión parcial o total del tratamiento farmacológico. ${ }^{17-19}$ Algunos estudios sugieren que el efecto de la inmunoterapia puede perdurar durante varios años luego de su suspensión si se hace el ciclo completo (3 a 5 años); sin embargo, la mayor parte de esos estudios se efectuaron en pacientes sensibilizados a granos de polen con rinitis ${ }^{20,21}$ y poco se sabe si este efecto se reproduce en pacientes sensibilizados a ácaros, principal 
fuente de alergenos en el trópico y con síntomas respiratorios más severos, como el asma.

En este artículo se evalúa, como desenlace primario, el efecto clínico de la inmunoterapia en pacientes con asma sensibilizados a ácaros, dos años después de haber terminado el tratamiento. Las variables utilizadas fueron: medición del número de exacerbaciones y la dosis de tratamiento farmacológico. Como desenlaces secundarios se evaluó si la edad de inicio a la inmunoterapia es un factor que influye en la evolución clínica.

\section{MATERIAL Y MÉTODO}

Estudio longitudinal, ambispectivo, abierto, no intervencionista, conformado por una cohorte de pacientes con diagnóstico de asma que asistieron de septiembre de 2009 a enero de 2012 al centro de salud de la Universidad de Antioquia (Medellín, Colombia). A partir de esta población se formaron dos grupos según si recibieron solo tratamiento con fármacos (control) o, además, inmunoterapia subcutánea con ácaros Der f, Der p y/o Blot (activo). El esquema de inmunoterapia utilizado fue "ultra-rush" con una dosis subcutánea de $0.5 \mathrm{ml}$ de mantenimiento mensual. Debido a que este estudio fue no intervencionista, la administración o no de inmunoterapia se determinó según el criterio médico o la preferencia del paciente durante la consulta inicial. Para reducir los sesgos al momento de la selección, por cada paciente que fue admitido al grupo de inmunoterapia, se incluyeron, al menos, dos pacientes al grupo control, con características similares sociodemográficas y asociadas con la enfermedad (tiempo de inicio, severidad, tratamiento, etc.).

Para la evaluación del desenlace primario como variables de medición se usó el número de exacerbaciones y el tratamiento farmacológico en relación con la dosis día. Para evaluar el desenlace secundario se efectuó un análisis estratificado, de acuerdo con la edad de los pacientes (menores o mayores de 14 años). Se escogió la edad de 14 años teniendo en cuenta los resultados observados en estudios previos en la población de estudio. ${ }^{22}$ Todos los pacientes recibieron tratamiento farmacológico de acuerdo con las recomendaciones de las guías GINA (www.ginasthma.org) con esteroides, antileucotrienos o beta-agonistas según la severidad, la progresión y el control de los síntomas. Debido a las diferencias en las dosis de esteroides y en el tratamiento según la edad de los pacientes, se hizo una comparación entre los grupos de edad según el porcentaje de cambio de la dosis en relación con el tiempo de administración. Debido a que el estudio no fue intervencionista y algunos datos eran retrospectivos, la severidad de las exacerbaciones no pudo medirse. Solo se incluyeron las que reportaron uso de salbutamol con esquema de rescate o la asistencia a urgencias, o ambas. El apego al tratamiento farmacológico y sus dosis también fue registrado.

\section{Criterios de selección}

Se incluyeron pacientes mayores de tres años de edad, con asma alérgica persistente de severidad moderada y sensibilización demostrada a ácaros Dermatophagoides farinae (Der f), D. pteronyssinus (Der p) o Blomia tropicalis (Blo t), cuyos síntomas respiratorios hubieran iniciado durante la primera década de la vida. Se excluyeron los pacientes (del grupo control y activo) que requirieran el uso permanente de inmunosupresores o que tuvieran contraindicación a los medicamentos convencionales para control del asma o la inmunoterapia. ${ }^{23}$ Tampoco se incluyeron al estudio los pacientes que hubieran recibido previamente algún agente biológico. Los pacientes con inmunoterapia debían haber tenido un cumplimiento anual al tratamiento de al menos $70 \%$ en cuanto al número de dosis administradas. 
Todos los pacientes tenían evaluación de la sensibilización a las fuentes de alérgenos más frecuentes en la población de estudio mediante pruebas intraepidérmicas o medición de IgE específica de acuerdo con los criterios del GA2LEN. ${ }^{24}$

\section{Consideraciones éticas}

El tratamiento farmacológico o inmunoterapia administrado a todos los pacientes siguió los lineamientos de las guías internacionales de tratamiento convencional del asma. Los datos que se reportan son resultado del seguimiento de los pacientes y la revisión sistemática de sus registros clínicos. Todos los pacientes, o en caso de ser menores de edad sus padres o tutores, firmaron un consentimiento informado para el inicio de la inmunoterapia validado por el comité de ética de la Universidad de Antioquia, que además evaluó y otorgó el permiso para la evaluación de los registros respetando el anonimato de los pacientes.

\section{Análisis}

Los análisis se realizaron con el programa de cómputo SPSS versión 21 para Windows con U de Wilcoxon para análisis intergrupos, Mann Whitney para intragrupos y la prueba $T$ para diferencias de media en muestras independientes. Una $p<0.05$ se consideró estadísticamente significativa. Debido a que el efecto de la inmunoterapia es detectable después de varios meses de su aplicación, los análisis se efectuaron con base en análisis por protocolo. Para el análisis de múltiples comparaciones se utilizó el test de Dunn. Las proporciones se analizaron con tablas de contingencias y prueba exacta de Fisher.

\section{RESULTADOS}

Durante el periodo de evaluación se revisaron 1014 historias clínicas. En el grupo control 122 pacientes y 384 en el grupo activo cumplieron con los criterios de inclusión y el periodo de evaluación. El principal motivo de no inclusión al estudio de un paciente fue la falta de datos en la historia clínica respecto de las variables de estudio (número de exacerbaciones, dosificación del tratamiento farmacológico, motivos de suspensión del tratamiento, etc.) (Figura 1). Las características de los pacientes excluidos fueron similares a las de los pacientes que permanecieron durante el seguimiento. La edad de los pacientes, severidad de los síntomas, la edad media al inicio del asma, y el tratamiento farmacológico fueron similares en ambos grupos. Todos los pacientes estaban sensibilizados a ácaros (Blot, Der $\mathrm{f}$ y/o Der p) y la frecuencia de sensibilización a mascotas fue superior al $30 \%$ (Cuadro 1).

\section{Evaluación del control de síntomas}

En la primera evaluación todos los pacientes habían tenido una o más exacerbaciones de asma al mes. En la Figura 2 se representa el total de exacerbaciones por año en cada grupo. En promedio, al iniciar el estudio cada paciente

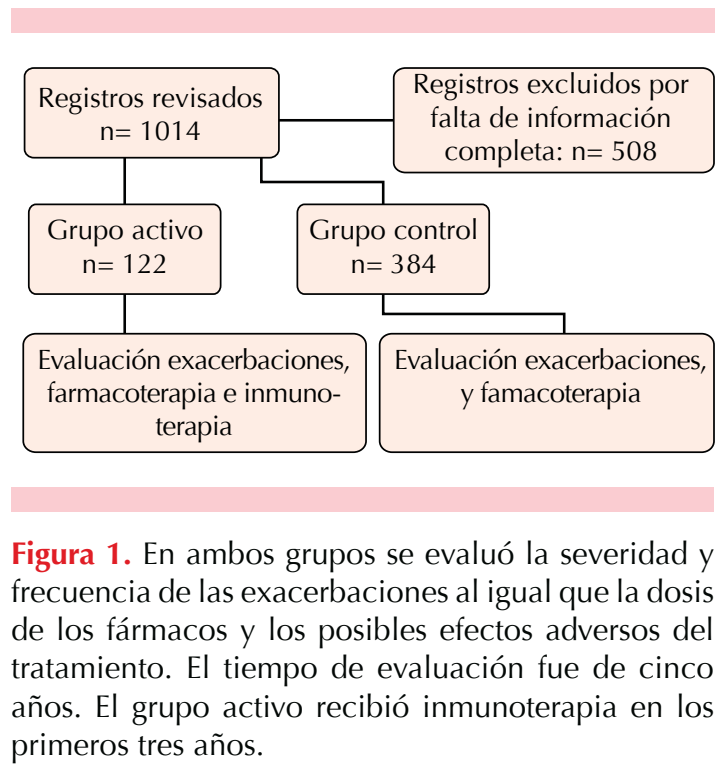


Cuadro 1. Características de los pacientes.

\begin{tabular}{lcc|}
\hline \multirow{2}{*}{ Características } & \multicolumn{2}{c}{ Grupo } \\
& Activo & Control \\
Número de pacientes & $122(100 \%)$ & $384(100 \%)$ \\
Edad & $8(3-49)$ & $9(3-38)$ \\
Sexo femenino & $63(51 \%)$ & $193(50 \%)$ \\
Sensibilización & $122(100 \%)$ & $384(100 \%)$ \\
$\quad$ Ácaros & $122(100 \%)$ & $384(100 \%)$ \\
$\quad$ Mascotas & $42(34 \%)$ & $136(35 \%)$ \\
Conjuntivitis & $104(86 \%)$ & $294(76 \%)$ \\
Rinitis & $94(77 \%)$ & $284(73 \%)$ \\
\end{tabular}

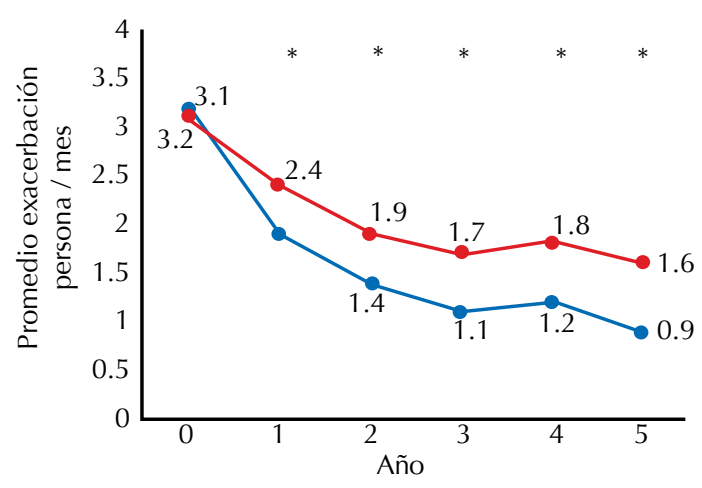

Figura 2. Número de exacerbaciones promedio por paciente al mes en los grupos activo (azul) y control (rojo). ${ }^{*} p<0.05$.

tenía tres exacerbaciones mensuales. En ambos grupos hubo una reducción significativa en la frecuencia de exacerbaciones de asma (Figura 2 ) luego del sexto mes $(p=0.04)$. En el grupo activo la disminución de las exacerbaciones fue superior al grupo control a partir del noveno mes $(p=0.05)$. Luego de trascurridos dos años de la suspensión de la inmunoterapia, no se observó una recaída significativa en el grupo activo y el número de exacerbaciones seguía siendo inferior que en el grupo control.

\section{Reducción del consumo de medicamentos}

Ambos grupos experimentaron una reducción importante de la medicación requerida para el control de los síntomas a partir del noveno mes y fue dependiente de la dosis de inhaladores. No se observaron diferencias en cuanto al consumo de anti-leucotrienos. Esta reducción fue significativamente mayor en el grupo activo (Figura 3). Al finalizar la inmunoterapia, 50 (40.9\%) pacientes habían suspendido la aplicación de inhaladores bronquiales (esteroides solos o combinados con beta-agonistas de acción larga) en comparación con $88(22.9 \%)$ del grupo control $(p=0.01)$ (Figura 4). No se observó una recaída significativa en el grupo activo después de la suspensión de la inmunoterapia (Figura 4). No hubo diferencias significativas entre los grupos en cuanto a pacientes que requirieron aumento del tratamiento a los tres años (activo 12\% vs control 16\%) o a los 5 años (3 vs $2 \%$ ) ni de forma acumulada (15 vs $18 \%$ ).

\section{Edad y sensibilización como factores pronósticos de la inmunoterapia}

Al hacer el análisis de los subgrupos en el grupo activo con base en la edad y número de sensi-

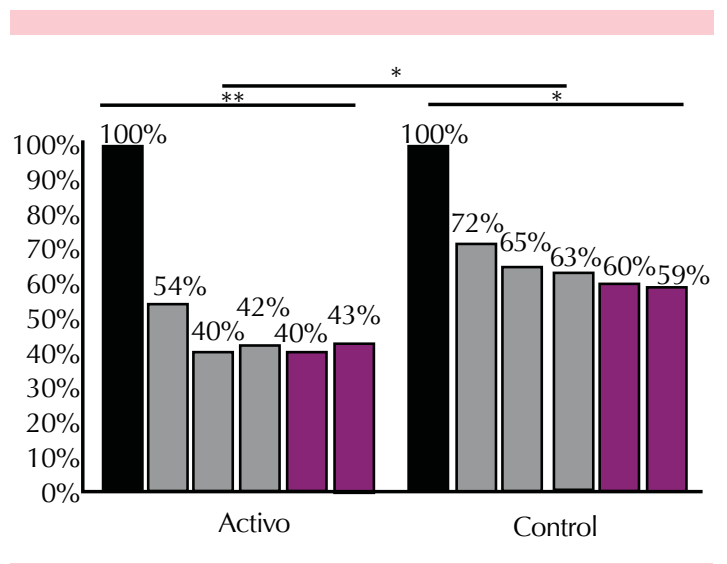

Figura 3. Reducción de medicamentos en el tiempo de seguimiento. El porcentaje representa el promedio de la dosis de inhaladores recibida en relación con la dosis administrada al inicio del estudio. La barra negra es el basal, las barras grises representan los tres primeros años y las moradas los dos años siguientes a la suspensión de la inmunoterapia en el grupo activo. * $p<0.05 * * p<0.01$. 


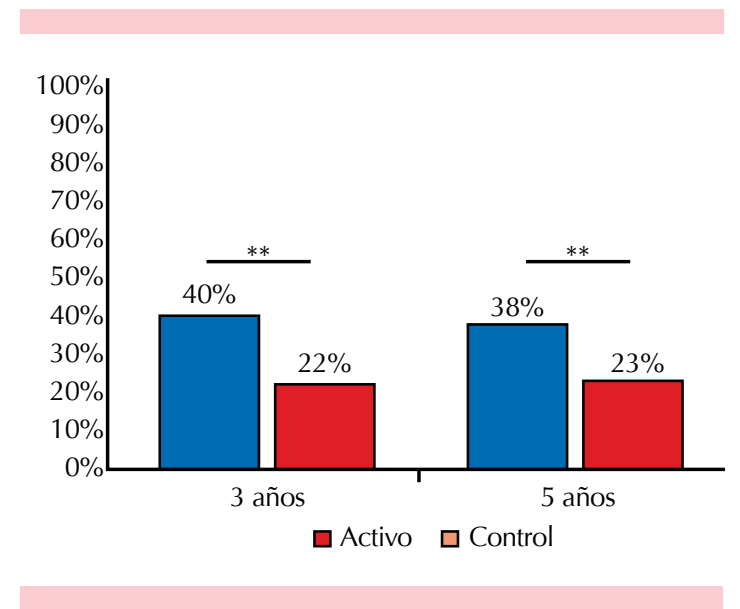

Figura 4. Suspensión total de inhaladores bronquiales a los 3 y 5 años. Grupo activo (azul) y grupo control (rojo). ${ }^{* *} p<0.01$.

bilizaciones se observó que los pacientes del grupo activo menores de 14 años tuvieron mayor reducción en la dosificación de los inhaladores y mayor suspensión total que los otros subgrupos; sobre todo cuando se compararon con el grupo polisensibilizado mayor de 14 años. En el Cuadro 2 se muestra la comparación de subgrupos en el grupo activo.

\section{Efectos adversos durante el seguimiento}

En 17 pacientes hubo 38 reacciones locales inmediatas (menos de una hora) después de la administración de la inmunoterapia, 23 de ellas en el transcurso de las 6 primeras dosis (una por mes). Las reacciones consistieron en eritema y prurito en el sitio de aplicación y remitieron en los primeros 60 minutos siguientes a la aparición. Se reportaron cuatro reacciones sistémicas que se controlaron durante la atención y con alta para los pacientes transcurridas 4 a 6 horas de observación, sin complicaciones tardías posteriores. Todos los pacientes continuaron con el tratamiento. La tasa de apego (número de citas para inmunoterapia cumplidas) fue de $76 \%$. Se reportaron de 58 reacciones locales en el área peribucal quizá asociadas con los esteroides inhalados en 49 pacientes. En todos los casos se consiguió la remisión de los síntomas y no fue necesario suspender el tratamiento.

\section{DISCUSIÓN}

Debido a la alta frecuencia y al carácter crónico del asma los costos del tratamiento son elevados para el paciente y el sistema de salud, con las molestias e inconvenientes que ello implica. Por esto el asma representa un problema de salud pública y una necesidad de identificar las formas de tratamiento que resulten más costo-efectivas para el paciente y los servicios públicos de salud.

Diversos estudios sustentan que la inmunoterapia es efectiva en el control del asma, lo que permite reducir la necesidad de medicamentos controladores, las asistencias a urgencias u hospitalizaciones y otros rubros derivados de la atención directa e indirecta de estos pacientes. ${ }^{11,19,23,25}$ Además, si es administrada a niños con rinitis alérgica, disminuye significativamente el riesgo de padecer asma, incluso luego de haber suspendido el tratamiento..$^{26-28} \mathrm{~A}$ pesar de estos resultados, en muchas ocasiones el inicio de este tratamiento no se ofrece en los sistemas de salud o su inicio es muchos años de la aparición de los síntomas, lo que hace que su efecto clínico disminuya. ${ }^{22}$

En este estudio se evaluó el control sintomático de un grupo de pacientes con asma durante los tres años del tratamiento con inmunoterapia y dos años posteriores a su suspensión. Se observó que un porcentaje importante de los pacientes del grupo activo y control tuvieron mejor respuesta clínica, con menos exacerbaciones y menor necesidad de medicamentos controladores. El efecto de estas dos variables de evaluación fue más pronunciado en el grupo que recibió inmunoterapia antes del primer año de administración. Este efecto clínico se mantuvo por espacio de dos años posteriores a la suspensión, lo que indica que es similar a lo previamente re- 
Sánchez J y col. Impacto de la inmunoterapia con alergenos después de su suspensión

Cuadro 2. Edad de los pacientes y patrón de sensibilización

\begin{tabular}{|c|c|c|c|c|c|}
\hline \multicolumn{6}{|c|}{ Grupo activo $(n=122)$} \\
\hline & Menores de & años $(\mathbf{n}=\mathbf{8 0})$ & Mayores de 1 & años $(n=42)$ & \multirow[b]{2}{*}{$\boldsymbol{P}$} \\
\hline & $M S<14(n=36)$ & PS $<14 \quad(n=54)$ & $M S>14 \quad(n=20)$ & PS $>14 \quad(n=22)$ & \\
\hline Aumento de inhaladores+ & $3 \%$ & $4 \%$ & $2 \%$ & $5 \%$ & 0.1 \\
\hline Reducción de inhaladores++ & $50 \%$ & $40 \%$ & $36 \%$ & $30 \%$ & 0.04 \\
\hline Suspensión de inhaladores++ & $21(17 \%)$ & $12(9 \%)$ & $8(6 \%)$ & $6(4 \%)$ & 0.03 \\
\hline
\end{tabular}

portado en rinitis. La inmunoterapia permite una inmunomodulación que perdura en el tiempo.

Las variables de evaluación utilizadas se relacionan con gastos económicos directos para el paciente (asistencia a urgencias, dosis de la medicación, suspensión de inhaladores, etc.), lo que permite explorar el efecto de la inmunoterapia en relación con su costo-efectividad. Los resultados de este estudio son similares a los encontrados en la revisión de artículos publicados en donde la mayor parte de las evaluaciones económicas concluyen que la inmunoterapia con alérgenos reduce los costos del tratamiento de las alergias, tanto desde la perspectiva del paciente como del tercer pagador (sistema de salud), ${ }^{29}$ sobre todo en pacientes con rinitis y asma. Además, es una medida costo-efectiva en comparación con el tratamiento convencional con fármacos, lo que es más evidente cuando esta relación se expresa como costo-efectividad incremental (ICER). ${ }^{30}$ La heterogeneidad en la metodología de los estudios y las poblaciones estudiadas limitan las generalizaciones de sus conclusiones.

La relación costo-efectividad de la inmunoterapia depende de la duración del beneficio clínico después de terminar el tratamiento y de los costos acumulados de la inmunoterapia y de los medicamentos. Al empezar la inmunoterapia los costos de tratamiento son mayores porque los de la inmunoterapia se añaden, pero no reemplazan al tratamiento convencional con fármacos. El punto de equilibrio se consigue cuando se reduce el número de medicamentos utilizados y cuando se termina la inmunoterapia. Estos puntos son variables pero, generalmente, el punto de equilibrio se da antes del segundo año y la suspensión de la inmunoterapia entre el tercero y quinto años después del inicio. Por esta razón los modelos económicos de inmunoterapia se proyectan a un horizonte temporal de más de cinco años. ${ }^{31,32}$

Si bien desde todas las perspectivas se coincide en que la inmunoterapia es costo-efectiva, ésta es mayor desde la del paciente, pues es quien asume los costos indirectos de la enfermedad (abstencionismo laboral o escolar del paciente y de su grupo social), que según algunos estudios puede representar la mayor parte de los gastos asociados con la enfermedad.

Puesto que las revisiones sistemáticas del tema se basan en estudios publicados en países de Europa y Estados Unidos es esperado que se analicen en su mayoría estudios de inmunoterapia para polen o árboles ${ }^{33}$ y son pocos los artículos incluidos que evalúan la repercusión económica de la inmunoterapia con ácaros en asma. ${ }^{34,35}$ Además, las conclusiones de los estudios fármaco-económicos pueden variar de un lugar a otro pues en términos más cuantitativos y específicos, la costo-efectividad depende del 
tipo de población; pueden variar el costo de la inmunoterapia y el de la productividad laboral perdida.

Es recomendable que en cada país se realice una valoración de la tecnología en términos de su situación social y económica, precisamente por esta razón nuestros resultados son importantes para Latinoamérica porque exploraran la repercusión de la inmunoterapia en una población con asma en condiciones sociodemográficas similares a las reportadas en las principales ciudades tropicales de la región y que tal vez se semejen mejor las realidades socioeconómicas de la región en comparación con los estudios provenientes de Estados Unidos o Europa.

En este estudio solo se incluyeron pacientes con alta sospecha clínica de síntomas al exponerse a los ácaros y con inicio de la enfermedad durante la primera década de la vida; así pudo conseguirse una muestra homogénea de pacientes con respecto a la historia clínica. Al incluir un grupo control pudieron compararse las diferencias en la respuesta clínica secundarias a la inmunoterapia o al tratamiento farmacológico y hacer análisis de subgrupos según la edad y el estado de sensibilización. Similar a una observación previamente reportada por nuestro grupo $^{22}$ observamos que la edad de inicio de la inmunoterapia y el número de sensibilizaciones influye en la respuesta clínica al tratamiento inmunomodulador. La mejoría clínica encontrada en los pacientes polisensibilizados al administrar una sola fuente de alérgenos, indica que no es necesario administrar varias fuentes para obtener un efecto clínico, sino realizar una adecuada evaluación diagnóstica que permita identificar la fuente relevante en los síntomas del paciente. Sin embargo, es recomendable administrar el tratamiento de forma temprana para disminuir el número de nuevas sensibilizaciones. El mayor tiempo de exposición a fuentes de alérgenos produce un proceso inflamatorio más severo que se refleja en mayor número de sensibilizaciones y en tratamiento clínico más complejo.

Nuestro estudio tiene varias limitaciones secundarias a su diseño observacional y la ausencia de ciego. Entre las fortalezas está el largo tiempo de evaluación (5 años) y que al ser observacional, no intervencionista, permite apreciar de manera objetiva los cambios en los parámetros evaluados en el tiempo en la vida real. El uso de variables de evaluación objetivas permitió un seguimiento adecuado y confiable de los parámetros evaluados.

En resumen, observamos que la inmunoterapia es un tratamiento útil en los pacientes con asma de etiología alérgica y que sus efectos clínicos perduran más allá de su suspensión, al igual que confirmamos que la edad de inicio y el número de sensibilizaciones son factores decisivos en la repercusión clínica del tratamiento. Estos resultados pueden servir de punto de partida para estudios de costo-eficacia en la región del trópico que permitan establecer si la inmunoterapia debe ser implementada como política de salud.

\section{Agradecimientos}

En memoria de la Dra. Elizabeth López, quien colaboró activamente en la recolección y seguimiento de los pacientes.

Fuente de financiación:

Este trabajo fue financiado con recursos de la Universidad de Antioquia y de la IPS Universitaria de la Universidad de Antioquia.

\section{REFERENCIAS}

1. Dennis R, Caraballo L, Garcia E, Caballero A, Aristizabal G, Cordoba $\mathrm{H}$, et al. Asthma and other allergic conditions in 
Colombia: a study in 6 cities. Ann Allergy Asthma Immunol. 2004;93(6):568-74.

2. Vergara C, Caraballo L. Asthma mortality in Columbia. Ann Allergy Asthma Immunol. 1998;80(1):55-60.

3. Neffen H, Baena-Cagnani CE, Malka S, Sole D, Sepulveda $\mathrm{R}$, Caraballo L, et al. Asthma mortality in Latin America. J Investig Allergol Clin Immunol. 1997;7(4):249-53.

4. Eder W, Ege MJ, von Mutius E. The asthma epidemic. N Engl J Med. 2006;355(21):2226-35.

5. Anandan C, Nurmatov U, van Schayck O, Sheikh A. Is the prevalence of asthma declining? Systematic review of epidemiological studies. Allergy. 2010;65(2):152-67.

6. Caraballo L, Acevedo N. Allergy in the tropics: the impact of cross-reactivity between mites and ascaris. Front Biosci (Elite Ed). 2011;3:51-64.

7. Burbach G, Heinzerling L, Edenharter G, Bachert C, Bindslev-Jensen C, Bonini S, et al. GA(2)LEN skin test study II: clinical relevance of inhalant allergen sensitizations in Europe. Allergy. 2009;64(10):1507-15.

8. Sánchez J, Diez S, Cardona R. [Frequency of sensitization to animals in a tropical area]. Rev Alerg Mex. 2014;61(2):81-9.

9. Sanchez J, Diez S, Cardona R. Sensibilización a aeroalergenos en pacientes alérgicos de Medellín, Colombia Revista Alergia México. 2012;59(3):139-47.

10. Fasce L, Tosca MA, Baroffio M, Olcese R, Ciprandi G. Atopy in wheezing infants always starts with monosensitization. Allergy Asthma Proc. 2007;28(4):449-53.

11. Alzakar RH, Alsamarai AM. Efficacy of immunotherapy for treatment of allergic asthma in children. Allergy Asthma Proc. 2010;31(4):324-30.

12. Cox L. Allergen immunotherapy and asthma: efficacy, safety, and other considerations. Allergy Asthma Proc. 2008;29(6):580-9.

13. Cady C, Powell M, Harbeck R, Giclas P, Murphy J, Katial $R$, et al. IgG antibodies produced during subcutaneous allergen immunotherapy mediate inhibition of basophil activation via a mechanism involving both FcgammaRIIA and FcgammaRIIB. Immunol Lett. 2010;130(1-2):57-65.

14. Wing K, Sakaguchi S. Regulatory T cells as potential immunotherapy in allergy. Curr Opin Allergy Clin Immunol. 2006;6(6):482-8.

15. Pauli G, Larsen TH, Rak S, Horak F, Pastorello E, Valenta R, et al. Efficacy of recombinant birch pollen vaccine for the treatment of birch-allergic rhinoconjunctivitis. J Allergy Clin Immunol. 2008;122(5):951-60.

16. Copenhaver CC, Parker A, Patch S. Systemic reactions with aeroallergen cluster immunotherapy in a clinical practice. Ann Allergy Asthma Immunol. 2011;107(5):441-7.

17. Penagos M, Passalacqua G, Compalati E, Baena-Cagnani $\mathrm{CE}$, Orozco S, Pedroza A, et al. Metaanalysis of the efficacy of sublingual immunotherapy in the treatment of allergic asthma in pediatric patients, 3 to 18 years of age. Chest. 2008;133(3):599-609.
18. Compalati E, Passalacqua G, Bonini M, Canonica GW. The efficacy of sublingual immunotherapy for house dust mites respiratory allergy: results of a GA2LEN meta-analysis. Allergy. 2009;64(11):1570-9.

19. Abramson MJ, Puy RM, Weiner JM. Injection allergen immunotherapy for asthma. Cochrane Database Syst Rev. 2010(8):CD001186.

20. Jacobsen L, Niggemann B, Dreborg S, Ferdousi HA, Halken $S, H \varnothing s t A$, et al. Specific immunotherapy has long-term preventive effect of seasonal and perennial asthma: 10year follow-up on the PAT study. Allergy. 2007;62(8):943-8.

21. Möller C, Dreborg S, Ferdousi HA, Halken S, Høst A, Jacobsen $L$, et al. Pollen immunotherapy reduces the development of asthma in children with seasonal rhinoconjunctivitis (the PAT-study). J Allergy Clin Immunol. 2002;109(2):251-6.

22. Sánchez J, Restrepo M, Diez S, Cardona R. Comparación del efecto clínico de la inmunoterapia en pacientes con asma alérgica según la edad y el patrón de sensibilización. Alergia, Asma e Inmunología Pediátricas. 2014;23(1):6-14.

23. Bousquet J, Lockey R, Malling HJ. Allergen immunotherapy: therapeutic vaccines for allergic diseases. A WHO position paper. J Allergy Clin Immunol. 1998;102(4 Pt 1):558-62.

24. Heinzerling LM, Burbach GJ, Edenharter G, Bachert $C$, Bindslev-Jensen C, Bonini S, et al. GA(2)LEN skin test study I: GA(2)LEN harmonization of skin prick testing: novel sensitization patterns for inhalant allergens in Europe. Allergy. 2009;64(10):1498-506.

25. Zielen S, Kardos P, Madonini E. Steroid-sparing effects with allergen-specific immunotherapy in children with asthma: a randomized controlled trial. J Allergy Clin Immunol. 2010;126(5):942-9.

26. Kwon YS, Oh SH, Wu WH, Bae BG, Lee HJ, Lee MG, et al. CC chemokines as potential immunologic markers correlated with clinical improvement of atopic dermatitis patients by immunotherapy. Exp Dermatol. 2010;19(3):246-51.

27. Rossi RE, Monasterolo $G$, Incorvaia $C$, Moingeon $P$, Frati $F$, Passalacqua G, et al. Lack of neo-sensitization to Pen a 1 in patients treated with mite sublingual immunotherapy. Clin Mol Allergy. 2010;8:4.

28. Cantillo JF, Puerta L. [New approaches for allergen-specific immunotherapy]. Biomedica. 2010;30(3):440-53.

29. Simoens $S$. The cost-effectiveness of immunotherapy for respiratory allergy: a review. Allergy. 2012;67(9):1087-105.

30. Meadows A, Kaambwa B, Novielli N, Huissoon A, Fry-Smith $A$, Meads $C$, et al. A systematic review and economic evaluation of subcutaneous and sublingual allergen immunotherapy in adults and children with seasonal allergic rhinitis. Health Technology Assessment. 2013;17(27).

31. Bruggenjurgen B, Reinhold T, Brehler R, Laake E, Wiese G, Machate $U$, et al. Cost-effectiveness of specific subcutaneous immunotherapy in patients with allergic rhinitis and allergic asthma. Annals of allergy, asthma \& immunology: 
official publication of the American College of Allergy, Asthma, \& Immunology. 2008;101(3):316-24.

32. Hankin CS, Cox L, Bronstone A, Wang Z. Allergy immunotherapy: reduced health care costs in adults and children with allergic rhinitis. J Allergy Clin Immunol. 2013;131(4):1084-91.

33. Hankin CS, Cox L. Allergy immunotherapy: what is the evidence for cost saving? Curr Opin Allergy Clin Immunol. 2014;14(4):363-70.
34. Reinhold T, Ostermann J, Thum-Oltmer S, Bruggenjurgen B. Influence of subcutaneous specific immunotherapy on drug costs in children suffering from allergic asthma. Clinical and translational allergy. 2013;3(1):30.

35. Chen J, Xiang J, Wang Y, Shi Q, Tan H, Kong W. [Health economics analysis of specific immunotherapy in allergic rhinitis accompanied with asthma]. Lin chuang er bi yan hou tou jing wai ke za zhi = Journal of clinical otorhinolaryngology, head, and neck surgery. 2013;27(17):925-8. 